\title{
CPAP in fatty liver with obesity without topiramate and MOVE program does not cause sustained weight loss but may results in weight gain-an opinion!
}

Keywords: CPAP, OSA, phenteramine, topiramate, qsymia

\section{Opinion}

A recent study in this edition of this Journal by the author as well as one by Redenius and associates in Journal of Clinical Sleep Medicine have demonstrated that CPAP does not lead to change in BMI without the addition of topiramate and MOVE program. Starting in 2008 the Illinois Neurological Institute Sleep Center in Peoria Ill and University of Illinois College of Medicine at Peoria Il published a study of 183 subjects who had obesity with an average BMI of $35.9 \pm 10 \mathrm{~kg} / \mathrm{m}^{2}$ and a control group of 45 over 1 year period of CPAP for obstructive sleep apnea. Control subjects had no treatment. In the treatment group $53.0 \%(97)$ of subjects or lost $\geq 1 \mathrm{~kg} / \mathrm{m}^{2}$. In the control group, $55.6 \%(25)$ of subjects gained or lost $\geq 1 \mathrm{~kg} / \mathrm{m}^{2}$. The change in BMI between treatment group and controls did not differ significantly after 1 year. Anecdotally, some of the subjects according to the authors initially lost weight after starting CPAP but eventually gained it back 1 year later. ${ }^{1}$

Another published study of CPAP, Zaher Fanari reported on the impact of CPAP, weight loss or both on the metabolic syndrome profile in patients with OSA. 181 patients with obesity and C-reactive protein (CRP) greater than $1.0 \mathrm{mg} /$ liter were randomized to CPAP, a weight-loss intervention, or CPAP plus weight loss intervention for 24 weeks. The results in 146 participants with a reduction in CRP levels, insulin resistance and serum triglyceride levels in those assigned to weight loss only and to the combined interventions, but not in the CPAP alone group. BMI did not significantly decrease in any group treated with CPAP. ${ }^{2}$

Kajaste et al. ${ }^{3}$ published a study of 2 years weight reduction for 31 obese male sleep apneics. Between CPAP group and controls with a weight loss program and no CPAP, There was no significant weight difference between the two groups, although both had lost weight irrespective of CPAP use. The Kajaste study relied on self- reporting of CPAP and the authors believed poor adherence to CPAP treatment may have been responsible for the lack of difference between the two groups. ${ }^{3}$

Winslow, Bowden, Didonato and McCullough in 2012 used phenteramine $15 \mathrm{mg}$ plus extended release topiramate $92 \mathrm{mg}$ in fortyfive subjects with moderate OSA not receiving CPAP with a BMI of $30-40 \mathrm{~kg} / \mathrm{m}^{2}$. Phentermine plus extended-release topiramate induced significant weight reductions and concomitant improvements in OSA and related symptoms vs placebo. This suggests weight loss mediated by phentermine plus extended-release topiramate may be useful in treatment of moderate to severe OSA in obese subjects unable or unwilling to comply with CPAP treatment. ${ }^{4}$

The present report in this edition of Sleep Medicine and Disorders
Volume I Issue 6 - 2017

\author{
Trent W Nichols \\ Gastroenterologist and Neuroscience, USA
}

Correspondence: Trent W Nichols, MD, CNDD and Quietmind Foundation, 72I Ash Dr, Hanover PA I733I, USA, Tel 717-633-5540,Email twnicholpa@comcast.net

Received: December 21, 2017 | Published: December 29, 2017

of 28 veterans in a VA clinic with fatty liver demonstrates weight loss in 28 subjects with OSA. All patients were treated with CPAP, a MOVE program combined with topiramate, and dietary consult in an observation study reported this month. Additionally the mitochondrial defect in obesity and sleep medicine as directed energy is discussed. ${ }^{5}$

Topiramate which has been used in epilepsy is thought by experts in neuroscience that it enhances networks in the brain that have a calming effect and inhibits networks that are over stimulating. "That seems to reduce food cravings," says Joel Yager, MD, a professor of psychiatry at the University of Colorado in Denver, who notes that the drug is also used to combat cravings for alcohol and cigarettes. Topramax (topiramate) has been reported to be effective in eating disorders recently. "Topiramate is most effective for people who are overweight or obese with binge-eating disorder or bulimia nervosa, and the additional benefit is that they can lose some weight," he says. "But it's not a first-line drug because a significant minority of patients will have bothersome, and sometimes serious, side effects. Also, many people with bulimia nervosa are flirting with anorexia nervosa, and topiramate may cause unwanted additional weight loss." Studies have been focused on topiramate for binge-eating disorder, and a few have examined its use in treating bulimia nervosa. Most were lasted a short period of time (12 to 24 weeks), included primarily women, and excluded people with psychiatric problems, which are common among people with eating disorders. Most studies also noted a high placebo response. In addition, pharmaceutical companies that market topiramate sponsored nearly all of the significant published trials, raising the possibility of a conflict of interest. ${ }^{6}$

However in the 2007 study by Brazilian researchers of 73 obese binge eaters were randomly assigned to take topiramate or a placebo in addition to participating in cognitive behavioral therapy (CBT), the ones who took topiramate plus CBT experienced greater weight loss and a higher rate of abstention from binging during treatment. ${ }^{7}$

Topiramate and phentermine in an extended release has been approved for weight reduction by the FDA since July 2012 called Qsymia. ${ }^{8}$ Topiramate when given at bedtime in patients with sleep 
apnea and obesity has the desired side effect of nocturnal sedation that was especially helpful for those with "mask anxiety" for those undergoing CPAP for the first time.

\section{Acknowledgements}

None.

\section{Conflicts of interest}

The author declared that there are no conflicts of interest.

\section{References}

1. Redenius R, Murphy C, O'Neill E, et al. Does CPAP lead to change in BMI? J Clin Sleep Med. 2008;4(3):205-209.

2. Fanari Z. The impact of CPAP, weight loss, or both on the metabolic syndrome profile in patients with OSA. $N$ Engl $J$ Med. 2014;370:22652275 .

3. Kajaste S, Brander PE, Telakivi T, et al. A cognitive-behavioral weight reduction program in the treatment of obstructive sleep apnea syndrome with or without initial nasal CPAP: a randomized study. Sleep Med. 2004;5(2):125-131
4. Winslow DH, Bowden $\mathrm{CH}$, Di Donato $\mathrm{KP}$, et al. A randomized, doubleblind, placebo-controlled study of an oral, extended-release formulation of Phenteramine/topiramate for the treatment of obstructive sleep apnea in obese adults. Sleep. 2012;35(11):1529-1539.

5. Nichols TW. Mitochondrial dysfunction in our aging veterans; Obesity, fatty liver, and NASH with obstructive sleep apnea treated with CPAP, medication, nutrition and MOVE program. SMDIJ; 2017

6. www.consumerreports.org/cro/2012/04/eating-disorders-may-be-treatedby-topamax/index.htm

7. Munsch S, Biedert E, Meyer A, et al. Randomized comparison of cognitive behavioral therapy and behavioral weight loss treatment for overweight individuals with binge eating disorder. Int J Eat Disord. 2007;40(2):102113.

8. Qsymia (phentermine and topiramate extended-release). Mountain View USA: FDA; 2014. 